\title{
Effects of Nitrites on Arterial Collateral Vessels of Dogs
}

\author{
Juro Irivahijima, M.D.
}

\begin{abstract}
SUMMARY
In dogs weighing about $10 \mathrm{Kg}$. anesthetized with pentobarbital, resistances of the collateral flow channels of the common carotid and femoral arteries were studied during occlusion of these arteries. When the common carotid artery was occluded, collateral resistance gradually decreased in about $30 \mathrm{sec}$., from initial collateral resistance (ICR) of $0.842 \pm 0.165$ $\mathrm{mm} . \mathrm{Hg} / \mathrm{ml} . / \mathrm{min}$. (mean with SE from 6 dogs) to steady state collateral resistance $(\mathrm{SCR})$ of $0.519 \pm 0.111(-39.4 \pm 3.6 \%$, significant at $\mathrm{P}<0.001)$. Intravenous injection of a long-active nitrite, N-ethoxy-carbonyl-3morpholinosydnonimine (SIN-10), at a dose of $1 \mathrm{mg}$./Kg., diminished ICR $(-37.5 \pm 5.3 \%, n=6, P<0.001,15$ min. after administration) but not SCR, thus ICR approached SCR. This effect was marked 5-60 min. after the intravenous injection. ICR of the femoral artery was also decreased by SIN-10 $(-48.7 \pm 5.8 \%, n=6$, significant at $\mathrm{P}<0.001)$. Nitroglycerin had a similar effect of dilating the collateral vessels for both arteries, though its effect was more evanescent in the time course and almost disappeared $5 \mathrm{~min}$. after injection. It is concluded that nitrites dilate almost the same collateral vasculature which would gradually open during arterial occlusion without nitrites.
\end{abstract}

Additional Indexing Words :

Arterial occlusion Collateral resistance Collateral flow Arterioarterial anastomosis Nitroglycerin

WHEN an artery is occluded, unless it is an end artery, the vascular area which has been supplied by that artery will receive blood supply from adjacent arteries through collateral vessels or arterioarterial anastomoses. In general collateral vessels are thinner and more resistant to flow than the original artery. Therefore, a large pressure drop is caused when blood flows through collateral vessels. Resistance of collateral vessels can be calculated from measurements of pressure and flow. ${ }^{11-3)}$ This method enables one to quantitate the changes in collateral vessels in response to various stimuli. In the present study, effects of two nitrites, N-ethoxy-carbonyl-3-morpholinosydnonimine (SIN-10) and nitroglycerin, on collateral resistances for the carotid and femoral arteries of the dog were studied.

From the Department of Physiology, Faculty of Medicine, University of Tokyo, Tokyo. Received for publication August 30, 1971. 


\section{Methods}

Male and female mongrel dogs weighing about $10 \mathrm{Kg}$. were anesthetized with an intravenous injection of $30 \mathrm{mg} . / \mathrm{Kg}$. of pentobarbital sodium. As a maintenance dose $1.5-3 \mathrm{mg} . / \mathrm{Kg}$. were added at approximately 1 -hr. intervals. Flow and pressure measurements were delayed until $10 \mathrm{~min}$. passed after each addition of the anesthetic.

The left common carotid artery was exposed at the neck for about $5 \mathrm{~cm}$. A 2.5 or $3 \mathrm{~mm}$. Statham electromagnetic flow probe was placed around the artery. Polyethylene tubes were inserted into the left brachial and left lingual arteries and connected to strain-gauge pressure transducers to record systemic and intrasinus pressures. Prior to the carotid occlusion experiment, the ipsilateral carotid sinus nerve was cut to minimize the change in systemic arterial pressure during occlusion. For femoral flow, the left femoral artery was exposed in the inguinal region and a 2 or $2.5 \mathrm{~mm}$. How probe was attached. Occlusion of each artery was done by applying a serrafine clamp distal to the flow probe. Pressure in the femoral artery distal to the occlusion was measured by cannulating the first side branch from the probe. Flow and systemic arterial pressure signals were smoothed to observe mean values, while pressure distal to the site of occlusion was recorded without intentional smoothing. All flow and pressures were recorded simultaneously with a penwriting oscillograph.

N-ethoxy-carbonyl-3-morpholinosydnonimine (SIN-10) is a newly synthetized, long-active nitrite with a potent hypotensive effect, mainly due to a direct action on vascular beds, especially on capacitance vessels. ${ }^{4,5)}$ This substance was dissolved in saline at a concentration of $1 \mathrm{mg} . / \mathrm{ml}$. for intravenous injection. Nitroglycerin was also dissolved in saline so that each $\mathrm{ml}$. of the solution contained $150 \mu \mathrm{g}$. of this drug. Throughout the study, a dosage of $1 \mathrm{mg} . / \mathrm{Kg}$. was routinely used for SIN-10 and $15 \mu \mathrm{g} . / \mathrm{Kg}$. for nitroglycerin. These dosages were determined in preliminary experiments as that which would elicit a moderate response.

\section{RESULTS}

Decrease in collateral resistance during arterial occlusion. An example of simultaneous recording of common carotid flow, systemic arterial pressure and intrasinus pressure is presented in Fig. 1, left panel. At the center of this recording, the common carotid artery was occluded for about $30 \mathrm{sec}$. During occlusion, intrasinus pressure which was distal to the occlusion was first dropped precipitously, then gradually recovered partially and reached a plateau in about $30 \mathrm{sec}$.

If collateral channels were entirely absent, the pressure distal to the occlusion would monotonically decrease to approach venous pressure. The considerably high distal pressure as seen in Fig. 1, left panel, was due to a well developed collateral circulation through the circle of Willis and other communications. ${ }^{6)-8}$ The collateral communication consists of many vessels, 

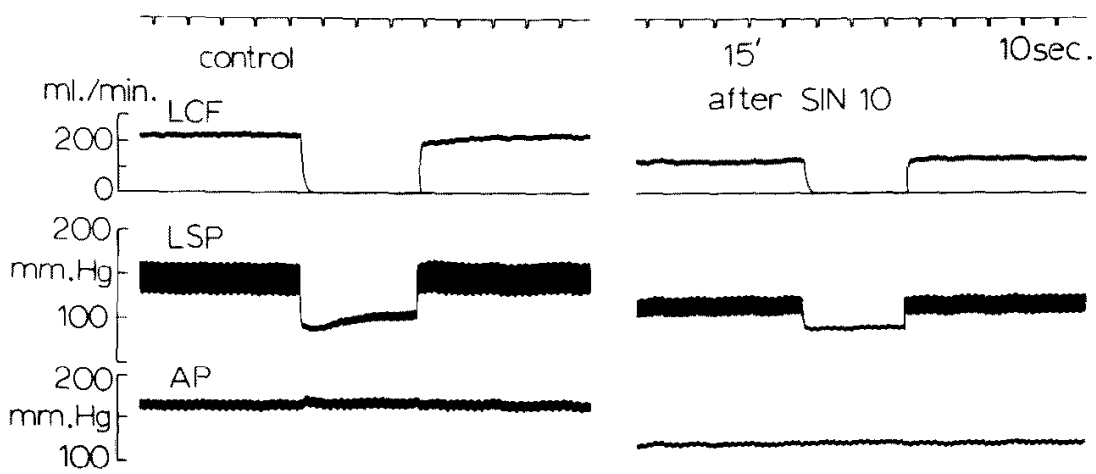

Fig. 1. Simultaneous recording of left carotid flow (LCF), left intrasinus pressure (LSP), and systemic arterial pressure (AP). The left carotid artery was occluded for about $35 \mathrm{sec}$. Left: control; Right: $15 \mathrm{~min}$. after intravenous injection of $S I N-10$ at a dose of $1 \mathrm{mg} \cdot / \mathrm{Kg}$.

presumably repeatedly branching and rejoining. However, we treated all these collateral vessels as a single vessel to which was assigned a single flow resistance $r$, the carotid collateral resistance. ${ }^{3}$ If we denote peripheral resistance of the entire area supplied by the common carotid artery by $\mathrm{R}$, it may be said that, when the artery is occluded, collateral flow first passes through $r$ and then through $R$ to flow out into the venous side. If we assume that intrasinus pressure is the pressure at the junction of $r$ and $R$, also assuming Poiseuille's law and venous pressure of zero, $P_{s}=P R /(r+R)$, where $P_{s}=$ intrasinus pressure and $\mathrm{P}=$ systemic arterial pressure. Thus $r=R\left(P-P_{s}\right) / P_{s}$. Assuming further that $\mathrm{R}$ remains unchanged during carotid occlusion, $\mathrm{R}$ may be computed as $P / i$, where $i=$ carotid flow before occlusion. The reason for the assumption that $\mathrm{R}$ is constant is that there was almost no reactive hyperemia after the reopening the carotid. Perhaps the collateral flow was sufficient to impede lack of oxygen ${ }^{2}$ and accumulation of vasodilator metabolites during occlusion. Since the carotid sinus was denervated, the change in vasomotor nerve activity by occlusion was thought to be minimal.

The partial recovery of intrasinus pressure $\left(P_{s}\right)$ during carotid occlusion indicated a decrease in $r$. In this study, the $r$ immediately after occlusion is referred to as initial collateral resistance (ICR) and the plateau value reached in about $30 \mathrm{sec}$. as steady state collateral resistance (SCR). For the experiment in Fig. 1, left panel, ICR was $0.43 \mathrm{~mm} . \mathrm{Hg} / \mathrm{ml} . / \mathrm{min}$. while SCR was $0.24 \mathrm{~mm} . \mathrm{Hg} / \mathrm{ml} . / \mathrm{min}$. In these and the following calculations the difference between systemic arterial pressure and intrasinus pressure before carotid occlusion was neglected and systemic pressure was assumed to remain unchanged during occlusion. The results of measurements of ICR and SCR in 6 dogs are summarized in Table I. 
Table I. Changes in Carotid Collateral Resistance and Other Parameters during Carotid Occlusion*

Initial collateral resistance (ICR)

Steady state collateral resistance (SCR)

$\%$ change in collateral resistance

Carotid peripheral resistance $(R)$

Mean arterial pressure (P)
$0.842 \pm 0.165 \mathrm{~mm} . \mathrm{Hg} / \mathrm{ml} . / \mathrm{min}$.

$0.519 \pm 0.111 \mathrm{~mm} . \mathrm{Hg} / \mathrm{ml} . / \mathrm{min}$.

$-39.4 \pm 3.6 \%$

$1.09 \pm 0.28 \mathrm{~mm} . \mathrm{Hg} / \mathrm{ml} . / \mathrm{min}$.

$153 \pm 4.0 \mathrm{~mm} . \mathrm{Hg}$

* Each value is mean with SE from 6 dogs. $R$ and $P$ are the preocclusion values which remained almost unchanged during occlusion.

\section{Effect of a sydnonimine compound on carolid ICR and SCR}

Fig. 1, right panel, shows a carotid occlusion experiment $15 \mathrm{~min}$. after intravenous injection of SIN-10, at a dose of $1 \mathrm{mg}$. $/ \mathrm{Kg}$. The most conspicuous change after administration of SIN-10 was, besides the decreases in arterial pressure and carotid flow, that intrasinus pressure did not tend to recover but remained almost unchanged during occlusion. Considering that arterial pressure and hence intrasinus pressure had been lowered by SIN-10, intrasinus pressure during occlusion was fairly high from the onset of occlusion. This finding suggested that the collateral vessels which would have dilated after arterial occlusion without SIN-10 had already dilated almost fully after administration of this substance. This was further supported by calculating the collateral resistance. For the experiment reproduced in Fig. 1, right panel, $\mathrm{ICR}=\mathrm{SCR}=0.25 \mathrm{~mm} . \mathrm{Hg} / \mathrm{ml} . / \mathrm{min}$. Peripheral resistance for this artery was $0.65 \mathrm{~mm} . \mathrm{Hg} / \mathrm{ml} . / \mathrm{min}$. before SIN-10 and 0.94 after SIN-10.

The above experiment was done in 6 dogs and the results are summarized in Fig. 2. Upon administration of SIN-10 (1 mg./Kg.), ICR was decreased to the level of SCR. This effect was maximum from $5 \mathrm{~min}$. to 1 hour after administration and gradually disappeared in the following hours. On the other hand, the change in SCR by SIN-10 was slight. Peripheral resistance of the vascular area supplied by the carotid artery $(R)$ was increased by SIN10. Since arterial pressure was decreased after administration of this substance, one might imagine that the increase in peripheral resistance was due to a reflex excitation of sympathetic vasoconstrictor fibers secondary to the arterial hypotension. However, a similar increase in peripheral resistance was observed after severance of the cervical sympathetics.

\section{Effect of nitroglycerin on carotid collateral resistance}

The experiment reproduced in Fig. 3 was done to observe the effect of nitroglycerin on the carotid collaterals. After a control occlusion experiment for the measurement of ICR and SCR, nitroglycerin was injected intravenously 
$\%(0.84: 0.17 \mathrm{~mm} . \mathrm{Hg} / \mathrm{ml} . \mathrm{min}$.
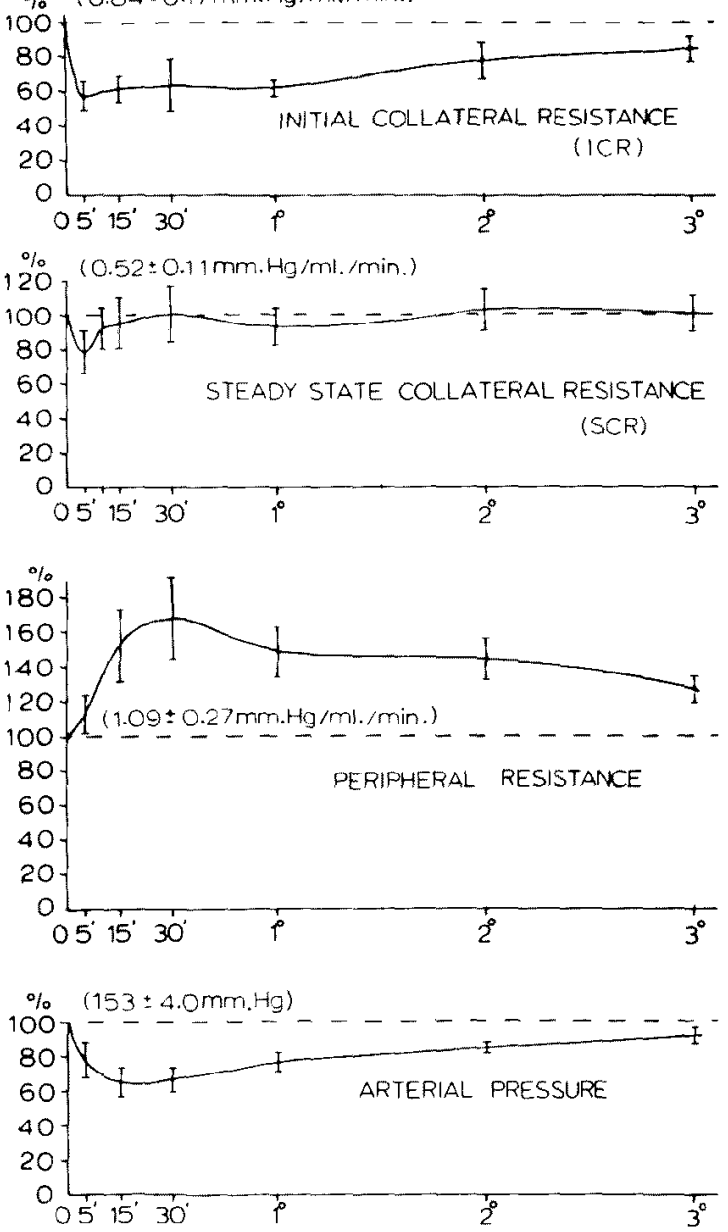

Fig. 2. Average changes ( $\pm \mathrm{SE}$ ) from 6 dogs of ICR, SCR, peripheral resistance and arterial pressure after intravenous injection of SIN-10 at 1 $\mathrm{mg} . / \mathrm{Kg}$. Percent of control values are plotted against time. Parentheses are control, pre-injection values.

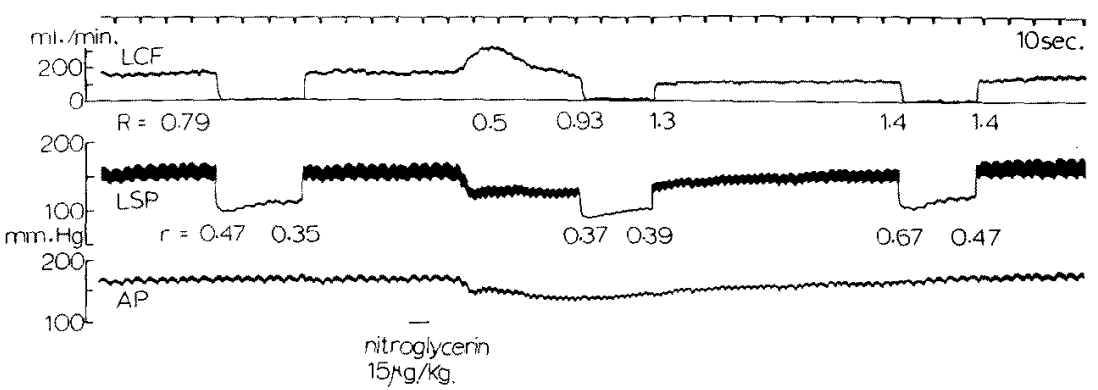

Fig. 3. Successive changes in carotid peripheral resistance (R) and carotid collateral resistance $(r)$ before and after intravenous injection of nitroglycerin $(15 \mu \mathrm{g} . / \mathrm{Kg}$.). Both resistances are in $\mathrm{mm} . \mathrm{Hg} / \mathrm{ml} / \mathrm{min}$. LCF : left carotid flow; LSP: left intrasinus pressure; AP: systemic arterial pressure. 

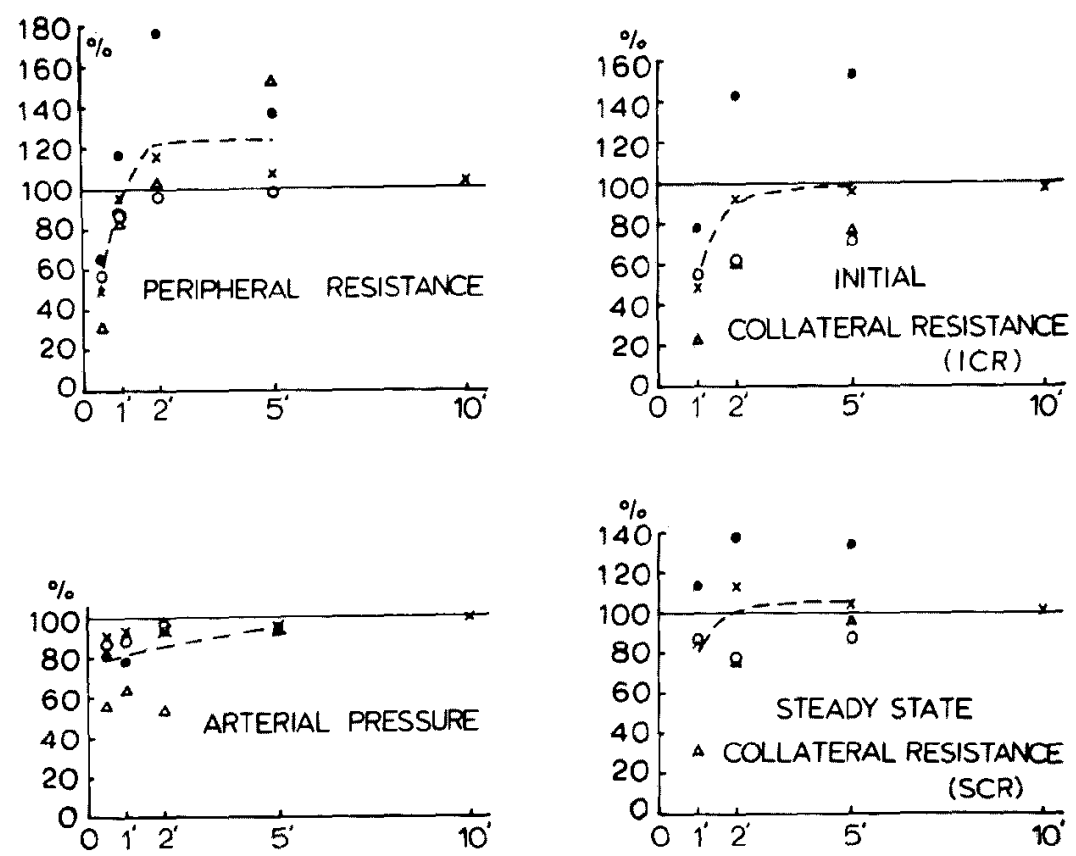

Fig. 4. Time coursc of percent changes of carotid peripheral resistance, systemic arterial pressure, initial collateral resistance (ICR) and steady state collateral resistance (SCR) after intravenous injection of nitroglycerin (15 $\mu \mathrm{g} . \mathrm{Kg}$.) in 4 dogs. Each symbol represents a different dog. Broken curves connect the mean values from the 4 dogs.

at a dose of $15 \mu \mathrm{g} . / \mathrm{Kg}$. The occlusion experiment which was performed 1 min. after the injection revealed a diminished ICR and an almost unchanged SCR. However, 3 min. after injection both ICR and SCR were higher than the control values in this particular instance. Peripheral resistance was diminished immediately (about $30 \mathrm{sec}$.) after injection, which was manifested by a marked rise of carotid flow in the face of a drastic lowering of arterial pressure. Such a decrease in carotid peripheral resistance was never observed with SIN-10. Later, however, peripheral resistance gradually increased to a level higher than the control even with nitroglycerin.

The results of the experiment with nitroglycerin in 4 dogs are plotted in Fig. 4. Like SIN-10, this nitrite decreased ICR but this effect was quite evanescent and recovery was almost complete in 2-5 min. The change in SCR was much smaller than that in ICR on administration of nitroglycerin. Peripheral resistance decreased markedly at first but then rapidly recovered and remained at a level higher than in the control for the period of 2-5 min. after injection. Arterial pressure recovered monotonically after the depressor effect of nitroglycerin. 


\section{Effect of nitrites on femoral collaterals}

Collateral vasculature is less developed for the femoral artery than for the common carotid artery. Therefore, the pressure distal to the occlusion dropped more severely when the femoral artery was occluded (Fig. 5, top). The distal pressure recovered gradually during occlusion, indicating a decrease in the collateral resistance also for this artery. ${ }^{1)}$ However, collateral flow did not seem to be sufficient to impede lack of oxygen and accumulation of vasodilator substances. A marked reactive hyperemia was observed after the reopening the artery. Therefore, peripheral resistance $(R)$ seemed to be decreased during arterial occlusion.

Since the constancy of $\mathrm{R}$ did not hold, it was impossible to calculate SCR for the femoral artery by the method employed for the carotid artery. However, it may be assumed that it takes several seconds for the vasodilator process to sufficiently develop in the area which was originally supplied by the occluded femoral artery. On this assumption, we can at least calculate ICR, since $R$ may be estimated to remain unchanged for the initial several seconds of the occlusion experiment.

As seen in Fig. 5, bottom, collateral resistance ( $r$ ) thus calculated was

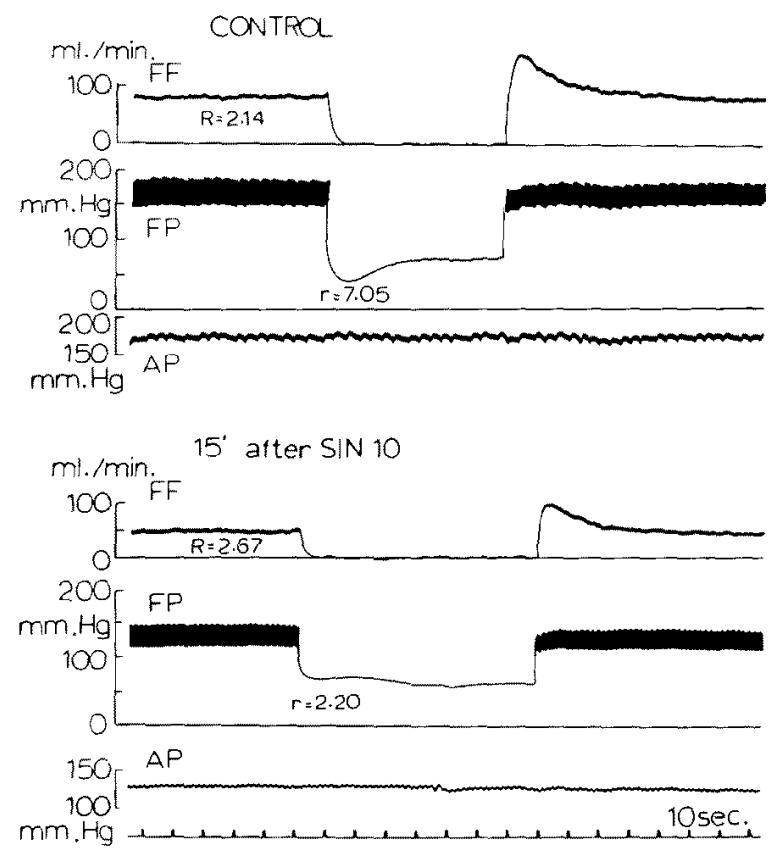

Fig. 5. Femoral artery occlusion experiment before (top) and $15 \mathrm{~min}$. after SIN-10 (1 mg./Kg.) (bottom). FF: femoral flow; FP: femoral pressure distal to the site of occlusion; AP: systemic arterial pressure. Note that, after SIN-10, distal pressure did not recover during occlusion but further decreased, suggesting gradual decrease in peripheral resistance with relatively constant collateral resistance. 

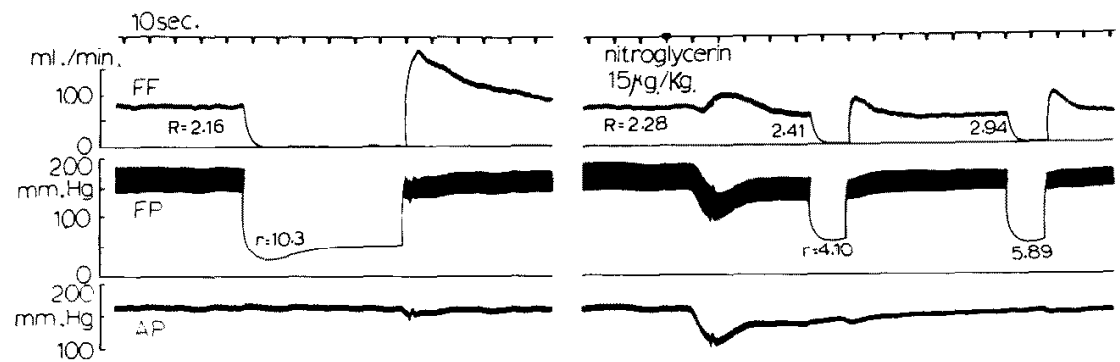

Fig. 6. Effects of nitroglycerin on femoral flow (FF), femoral pressure (FP), systemic arterial pressure (AP), femoral peripheral resistance (R) and femoral collateral resistance $(r)$. Left: control occlusion experiment before nitroglycerin.

greatly decreased by SIN-10. After administration of this substance, the pressure distal to the occlusion did not recover but was lowered further during the femoral artery occlusion. This indicated that peripheral resistance $(R)$ was diminished but collateral resistance ( $r$ ) was relatively unchanged. As also shown in Fig. 5, bottom, it took about $20 \mathrm{sec}$. for the distal femoral pressure to begin further decreasing. This finding supported the assumption made above that $\mathrm{R}$ remained unchanged until the vasodilator process had sufficiently developed during arterial occlusion. The decrease in femoral ICR by SIN-10 at a dose of $1 \mathrm{mg} . \mathrm{Kg}$., measured $15 \mathrm{~min}$. after injection, was $-48.7 \pm 5.8 \%$ (mean with $\mathrm{SE}$ from 6 dogs), significant at $\mathrm{P}<0.001$, while the change in peripheral resistance $(R)$ by $\mathrm{SIN}-10$ was insignificant $(-6.8 \pm 13.2 \%)$ for the femoral artery.

Femoral collateral resistance was also decreased by nitroglycerin as shown in Fig. 6. Similar to the carotid collateral resistance, after nitroglycerin, the decreased femoral collateral resistance recovered rapidly.

According to Rosenthal and Guyton, after occlusion of the femoral artery, it took about $12 \mathrm{~min}$. for the decreased femoral collateral resistance to fully recover. ${ }^{1)}$ In such an experiment as that reproduced in Fig, 6, 20 min. had passed after the control occlusion experiment before administration of nitroglycerin. Therefore, the decreased collateral resistance observed about 1 min. after injection was not due to the preceding occlusion experiment.

\section{Discussion}

Administration of SIN-10, a nitrite, induced a profound change in the course of distal pressure during arterial occlusion for both carotid and femoral arteries in the dog. Before this substance was given, the pressure distal to the occlusion, which dropped precipitously at first, gradually recovered partially 
due to dilatation of the collateral vessels. After SIN-10 the distal pressure did not recover during occlusion and, for the femoral artery, it was further decreased, presumably due to dilatation of the femoral resistance vessels. These observations of the pressure change pattern as well as the calculations of collateral resistances from pressure and flow data indicated that the collateral vessels for the carotid and femoral arteries, which were normally dilated after arterial occlusion due to ischemia and other vasodilating factors, seemed to be dilated without occlusion by SIN-10. Therefore, after this substance had been given, collateral resistance was not decreased further during arterial occlusion. A similar dilatation of collateral vessels was observed following administration of nitroglycerin, but the effect of this nitrite was more evanescent.

In the heart, it is said that collateral vasculaturc develops in areas of chronic myocardial ischemia. Such coronary collateral vessels dilate in response to nitrite administration, improving blood flow to the ischemic area. ${ }^{10}$ ) As far as the responsiveness to nitrites is concerned, a similarity is evident between the coronary collateral vessels and the carotid and femoral ones. Furthermore, the present study has also shown another similarity between the coronary and other vascular areas in that nitrites dilate relatively large vessels proximal to resistance vessels. The dilating effect of nitrites on such larger coronary vessels was described by Winbury and others. ${ }^{11}$ Therefore, the observation of changes in the collateral resistance of the carotid or femoral artery, introduced in this paper, might be useful in screening antianginal drugs.

\section{ACKNOWLEDGEMENT}

Thanks are due to the Takeda Chemical Co. for the generous supply of SIN-10.

\section{REFERENCES}

1. Rosenthal, S. L. and Guyton, A. C.: Circulat. Res. 23: $239,1968$.

2. Iriuchijima, J. and Koike, H.: Am. J. Physiol. 218: 876, 1970.

3. Iriuchijima, J., Koike, H., and Kurihara, M.: Pfügers Arch. 322: 304, 1971.

4. Kikuchi, K., Hirata, M., Nagaoka, A., and Aramaki, Y.: Jap. J. Pharmac. 20: 23, 1970.

5. Kikuchi, K., Hirata, M., and Nagaoka, A.: Jap. J. Pharmac. 20: 102, 1970.

6. Chungcharoen, D., DeBurgh Daly, M., Neil, E., and Schweitzer, A.: J. Physiol. 118: 509, 1952.

7. Jewell, P. A.: J. Anat. 86: 83, 1952.

8. Wang, S. C., Mazzella, H., and Heymans, C.: Arch. Intern. Pharmacodyn. 90: 1, 1952.

9. Guyton, A. C., Ross, J. M., Carrier, O., and Walker, J. R.: Circulat. Res. 14 \& 15: Suppl. I, $60,1964$.

10. Fam, W. M. and McGregor, M.: Circulat. Res. 18: 355, 1964.

11. Winbury, M. M., Howe, B. B., and Hefner, M. A.: J. Pharmacol. Exp. Therap. 168: 70, 1969. 